\title{
6 Asymptotic Expansions
}

\subsection{Asymptotic Series}

Physical problems often require a reasonably detailed knowledge of how particular functions behave at infinity. For example, if a function has an essential singularity at $z=\infty$ one may need a measure of how rapidly it blows up (or vanishes) as $z \rightarrow \infty$ along the real axis. Or, more detailed yet, one may actually have to evaluate the function for very large values of $|z|$. Recalling that few interesting functions are expressible in terms of the so-called elementary ones, this might appear to be a rather tall order to fill. Power series or infinite products are certainly unlikely to be helpful in most cases and integral representations would seem to offer a computational nightmare. Fortunately, the latter admit a property that obviates the need to explicitly evaluate them and hence, makes them an ideal starting point after all.

With only a modicum of manipulative effort, one can usually contrive to have an integral representation yield up an asymptotic expansion of the function it represents. In its simplest form an asymptotic expansion is a series in inverse powers of $z$ which, while not convergent, has partial sums that provide an arbitrarily good approximation, for sufficiently large $|z|$, of the function to which it corresponds. Thus, by conveying detailed information about the function's large $|z|$ behaviour, it meets our analytical needs to the letter.

The idea of approximating something by a divergent series may seem a little paradoxical. Therefore, we shall assist both our intuition and our credulity by running through a simple example.

Example: Consider the function of a real variable

$$
f(x) \equiv \int_{x}^{\infty} \frac{1}{t} e^{x-t} d t, \quad x>0 .
$$

By making the substitution $u=t-x$, we see that

$$
f(x)=\int_{0}^{\infty} \frac{e^{-u}}{u+x} d u<\frac{1}{x} \int_{0}^{\infty} e^{-u} d u=\frac{1}{x}, \quad x>0
$$

which immediately provides us with an upper limit on the values assumed by $f(x)$. To get a better idea of what these values may be, we now integrate (6.1.1) by parts. After $n$ such integrations, we find

$$
f(x)=\frac{1}{x}-\frac{1}{x^{2}}+\frac{2}{x^{3}}+\ldots+\frac{(-1)^{n-1}(n-1) !}{x^{n}}+(-1)^{n} n ! \int_{x}^{\infty} \frac{e^{x-t}}{t^{n+1}} d t .
$$


The form of this expression suggests that we examine the series

$$
\sum_{m=0}^{\infty} u_{m}(x), u_{m}(x)=\frac{(-1)^{m} m !}{x^{m+1}}
$$

Applying the ratio test, we have

$$
\lim _{n \rightarrow \infty}\left|\frac{u_{n+1}(x)}{u_{n}(x)}\right|=\lim _{n \rightarrow \infty} \frac{n}{x} \rightarrow \infty
$$

for fixed $x$. Thus, the series is divergent for all finite values of $x$. ference

This is not the set-back it might seem. Letting $S_{n}(x) \equiv \sum_{m=0}^{n} u_{m}(x)$, we find the dif-

$$
\left|f(x)-S_{n}(x)\right| \equiv R_{n}(x)=(n+1) ! \int_{x}^{\infty} \frac{e^{x-t}}{t^{n+2}} d t<(n+1) ! \int_{x}^{\infty} \frac{d t}{t^{n+2}}=\frac{n !}{x^{n+1}} .
$$

In other words, the error committed in approximating $f(x)$ by $S_{n}(x)$ is guaranteed to be less than $\frac{n !}{x^{n+1}}$. Thus, for fixed $n$, we can always find an $x$ sufficiently large to make this error less than any prescribed number $\varepsilon>0$. So, even though divergent, the series (6.1.3) has partial sums which provide an arbitrarily good approximation for $f(x)$, provided only that we restrict ourselves to sufficiently large values of $x$. Such a series is called an asymptotic series and its relationship to $f(x)$ is expressed formally by rewriting (6.1.2) to read

$$
f(x) \sim \sum_{m=0}^{\infty} \frac{(-1)^{m} m !}{x^{m+1}} .
$$

The symbol $\sim$ implies approximation rather than equality and in so doing takes cognizance of the divergent character of the series.

Notice that for a given value of $x$ there is a value of $n, N$ say, for which the upper bound on the error associated with the approximation is a minimum. Consequently, $N ! / x^{N+1}$ is a measure of the ultimate accuracy with which $f(x)$ can be computed. To estimate $N$ we note that

$$
\frac{n !}{x^{n+1}}<\frac{n^{n-1}}{x^{n+1}}=e^{(n-1) \ln n-(n+1) \ln x} .
$$

Differentiating the right hand side of this inequality with respect to $n$ and equating the result to zero, we find

$$
N \ln \frac{N}{\chi}+N=1 \quad \text { or, } \quad N \simeq x e^{(e / x-1)} .
$$

Thus, for $x=10, N=5$ which means that for this value of $x$, optimal accuracy is obtained with just the first five terms in the series. The associated error is then $\leq 0.00012$. 
Definition: A series $\sum_{m=0}^{\infty} c_{m} z^{-m}$, which either converges for large values of $|z|$ or diverges for all values of $z$, is said to be an asymptotic series for $f(z)$,

$$
f(z) \sim \sum_{m=0}^{\infty} c_{m} z^{-m},
$$

valid in a given range of values of $\arg z$, if, for any positive integer $n$,

$$
\lim _{|z| \rightarrow \infty}\left\{z^{n}\left[f(z)-\sum_{m=0}^{\infty} c_{m} z^{-m}\right]\right\}=0
$$

for $\arg z$ in this range.

An asymptotic series will be convergent only if the function it represents is holomorphic at $z=\infty$. Thus, more often than not, they are divergent series. As we have seen, this does not adversely affect their ability to accurately represent functions for large values of $|z|$. Since the difference between $f(z)$ and the first $(n+1)$ terms of the series (6.1.6),

$$
\left|f(z)-c_{0}-\frac{c_{1}}{z}-\ldots-\frac{c_{n}}{z^{n}}\right|,
$$

is of order $1 /|z|^{n+1}$, such a series is often better suited for numerical computation than a convergent representation would be. Some caution is necessary however. The addition of too many terms of a divergent series will render an approximation for fixed $z$ worse rather than better. Indeed, as we learned from our simple example, there is always an optimum number of terms that gives rise to the best approximation for any given value of $z$.

If a function $f(z)$ possesses an asymptotic series

$$
f(z) \sim c_{0}+\frac{c_{1}}{z}+\frac{c_{2}}{z^{2}}+\ldots,
$$

the series coefficients are uniquely determined by the equations

$$
\begin{aligned}
& \lim _{|z| \rightarrow \infty} f(z)=c_{0} \\
& \lim _{|z| \rightarrow \infty} z\left[f(z)-c_{0}\right]=c_{1} \\
& \lim _{|z| \rightarrow \infty} z^{2}\left[f(z)-c_{0}-\frac{c_{1}}{z}\right]=c_{2} \\
& \quad \ldots \\
& \lim _{|z| \rightarrow \infty} z^{n}\left[f(z)-c_{0}-\frac{c_{1}}{z}-\ldots-\frac{c_{n-1}}{z^{n-1}}\right]=c_{n} \\
& \ldots .
\end{aligned}
$$

This shows that a given function can only have one asymptotic series. However, knowledge of an asymptotic series does not determine a corresponding function since different functions can generate the same asymptotic series. For example, $e^{1 / z}$ and $e^{1 / z}+e^{-z}$ 
have the same asymptotic series

$$
1+\frac{1}{1 ! z}+\frac{1}{2 ! z^{2}}+\frac{1}{3 ! z^{3}}+\ldots
$$

valid in the range $|\arg z|<\pi / 2$.

Many functions $f(z)$ do not possess an asymptotic series; $e^{z}$ is an obvious example. However, even when this is the case, one can often find a second function $\varphi(z)$ such that the quotient $f(z) / \varphi(z)$ does possess a series,

$$
f(z) / \varphi(z) \sim c_{0}+\frac{c_{1}}{z}+\frac{c_{2}}{z^{2}}+\ldots,
$$

for some range of $\arg z$. For such functions we shall write

$$
f(z) \sim \varphi(z) \sum_{m=0}^{\infty} c_{m} z^{-m}
$$

and we shall use the term asymptotic expansion to refer interchangably to both a representation of this form as well as the more straightforward asymptotic series representation of (6.1.6). The term $c_{0} \varphi(z)$ in (6.1.9) is often called the dominant term of the expansion.

Example: The exponential integral function

$$
\operatorname{Ei}(x)=\int_{x}^{\infty} \frac{e^{-t}}{t} d t, \quad x>0
$$

differs from the function (6.1.1) of our first example by a factor of $e^{x}$. Thus, without further effort we deduce that $\operatorname{Ei}(x)$ has the asymptotic expansion $=$

$$
E i(x) \sim e^{-x}\left[\frac{1}{x}-\frac{1}{x^{2}}+\frac{2}{x^{3}}+\ldots+\frac{(-1)^{n-1}(n-1) !}{x^{n}}+\ldots\right] \sim \frac{e^{-x}}{x} \sum_{m=0}^{\infty} \frac{(-1)^{m} m !}{x^{m}} .
$$

Comparing this result with (6.1.9), we see that $\operatorname{Ei}(x)$ has $\frac{e^{-x}}{x}$ as its dominant term.

One can show that an asymptotic series can be added, multiplied and integrated term by term. However, it is not permissable in general to perform term by term differentiation.

To determine an asymptotic expansion one almost always proceeds from an integral representation of the function in which one is interested. This is due to two exceptional results: Watson's Lemma and the Method of Steepest Descents.

\subsection{Watson's Lemma}

George Neville Watson (1886-1965) was noted for the application of complex analysis to the theory of special functions. His collaboration while at Trinity College, Cambridge on 
the (1915) second edition of E. T. Whittaker's A Course of Modern Analysis (1902) produced an instant classic, a text that to this day is known simply as "Whittaker and Watson”. In 1922, he published a second classic text, Treatise on the Theory of Bessel Functions, which is an exhaustive study of all aspects of Bessel functions including especially their asymptotic expansions. Watson became Professor at the University of Birmingham in 1918, where he remained until 1951.

Watson's Lemma applies to functions $f(z)$ that can be represented by convergent integral transforms of the form

$$
f(z) \equiv \int_{0}^{\infty} e^{-z t} g(t) d t
$$

Although this makes it somewhat exclusive, it still covers many of the cases which occur in practice. All we require is that the spectral function $g(t)$ be holomorphic, except possibly for a branch-point at the origin, in the disc $|t|<T$ and admit the series representation

$$
g(t)=\sum_{m=1}^{\infty} c_{m} t^{m / p-1}, \quad|t| \leqslant \tau<T
$$

for some $p>0$. Also, let us suppose that when $t$ is real and positive and $t>T,|g(t)|<$ $K e^{b t}, K>0, b>0$. One can then show that term by term integration of the series together with the result

$$
\int_{0}^{\infty} e^{-z t} t^{m / p-1} d t=\frac{1}{z^{m / p}} \int_{0}^{\infty} e^{-u} u^{m / p-1} d u=\frac{\Gamma(m / p)}{z^{m / p}},
$$

yields the asymptotic expansion

$$
f(z) \sim \sum_{m=1}^{\infty} c_{m} \Gamma(m / p) z^{-m / p}
$$

for $|\arg z| \leqslant \pi / 2-\varepsilon$, where $\varepsilon$ is an arbitrary positive number.

Example: To illustrate the use of the lemma, let us return to the function featured in the first example of the previous Section,

$$
f(x)=\int_{x}^{\infty} \frac{e^{x-t}}{t} d t
$$

If we let $v=\frac{1}{x}(t-x)$, we can express $f(x)$ as a transform of the appropriate type,

$$
f(x)=\int_{0}^{\infty} e^{-x v} \frac{1}{v+1} d v .
$$


Moreover,

$$
\frac{1}{v+1}=\sum_{m=0}^{\infty}(-v)^{m}=\sum_{m=1}^{\infty}(-v)^{m-1}, \quad|v|<1 .
$$

Therefore, using (6.2.4), we immediately obtain the by now familiar asymptotic series

$$
f(x) \sim \sum_{m=1}^{\infty}(-1)^{m-1} \Gamma(m) x^{-m}=\sum_{m=1}^{\infty}(-1)^{m-1}(m-1) ! x^{-m} .
$$

Similarly, the complimentary error function

$$
\operatorname{erfc}(x)=1-\operatorname{erf}(x)=\frac{2}{\sqrt{\pi}} \int_{x}^{\infty} e^{-t^{2}} d t
$$

can be expressed as

$$
\operatorname{erfc}(x)=\frac{2}{\sqrt{\pi}} e^{-x^{2}} \int_{x}^{\infty} e^{-\left(t^{2}-x^{2}\right)} d t=\frac{2}{\sqrt{\pi}} e^{-x^{2}} \frac{x}{2} \int_{0}^{\infty} e^{-x^{2} v}[1+v]^{-1 / 2} d v
$$

where we have set $x^{2} v=t^{2}-x^{2}$. Now,

$$
\begin{aligned}
(1-v)^{-1 / 2} & =1-\frac{v}{2}+\frac{3 v^{2}}{8}-\frac{5 v^{3}}{16}+\frac{35 v^{4}}{128}-+\ldots \\
& =\sum_{m=1}^{\infty} \frac{(-1)^{m-1}(2 m-3) ! !}{2^{m-1}(m-1) !} v^{m-1}, \quad|v|<1 .
\end{aligned}
$$

Thus, using (6.2.4), we have

$$
\begin{aligned}
\operatorname{erfc}(x) & \sim \frac{2}{\sqrt{\pi}} e^{-x^{2}} \frac{x}{2} \sum_{m=1}^{\infty} \frac{(-1)^{m-1}(2 m-3) ! !}{2^{m-1}} x^{-2 m} \\
& \sim \frac{2}{\sqrt{\pi}} e^{-x^{2}} \sum_{m=1}^{\infty} \frac{(-1)^{m-1}(2 m-3) ! !}{2^{m}} x^{-(2 m-1)} .
\end{aligned}
$$

The method of steepest descent (which is also known as the saddle point method) provides a much more general technique for generating asymptotic expansions. Such generality does not come about without some cost however; this method is a good deal more complicated than is Watson's Lemma.

\subsection{The Method of Steepest Descent}

Consider the integral

$$
F(z)=\int_{C} e^{z f(t)} g(t) d t
$$


where $f(t), g(t)$ and $C$ have all been chosen so that the integrand is holomorphic in some domain containing $C$ and goes to zero at either end-point of $C$. It is entirely reasonable to expect that the most significant contributions to $F(z)$, for large values of $|z|$, arise from those segments of $C$ on which the real part of $[z f(t)]$ is large and positive. However, we must be mindful of the fact that the imaginary part of $[z f(t)]$ will generally increase as $|z|$ increases and that this will result in rapid oscillations of the factor $e^{i \operatorname{Im}[z f(t)]}$ and hence, in a complicated pattern of cancellations among the values assumed by the integrand. Such cancellations could make the evaluation of $F(z)$ a daunting if not impossible undertaking. However, we know that the integral is path independent within the integrand's domain of holomorphy. Therefore, we shall assume that this domain permits deformation of the contour of integration $C$ into a new contour $C_{0}$ on which $\operatorname{Im}[z f(t)]$ is constant whenever $\operatorname{Re}[z f(t)]$ assumes its largest values. Our first task then is to define $C_{0}$.

Since we want $\operatorname{Re}[z f(t)]$ to be large, we shall require $C_{0}$ to pass through a point $t=t_{0}$ at which $\operatorname{Re}[z f(t)]$ has a relative maximum and hence, at which

$$
\left.f^{\prime}\left(t_{0}\right) \equiv \frac{d f(t)}{d t}\right|_{t=t_{0}}=0
$$

As we shall see, we now need only demand that near $t=t_{0}$

$$
\operatorname{Im}[z f(t)]=\operatorname{Im}\left[z f\left(t_{0}\right)\right]
$$

to complete our specification of $C_{0}$.

We have already proven (the maximum modulus principle) that neither the real nor the imaginary part of a function can have an absolute maximum or minimum within its domain of holomorphy. However, we have yet to determine what does happen to a holomorphic function at a point where its first derivative vanishes. To do so now, let $w(x, y) \equiv u(x, y)+i v(x, y)$ be a holomorphic function whose derivative $\frac{d w}{d z}=0$ at some point $z=z_{0} \equiv\left(x_{0}, y_{0}\right)$. Clearly, the first partial derivatives of both $u(x, y)$ and $v(x, y)$ vanish at $\left(x_{0}, y_{0}\right)$. Moreover, since $u(x, y)$ and $v(x, y)$ are harmonic, $\nabla^{2} u=0$ and $\nabla^{2} v=0$, we know that if, for example, $\frac{\partial^{2} u}{\partial x^{2}}<0$ at $\left(x_{0}, y_{0}\right)$, then $\frac{\partial^{2} u}{\partial^{2} y}>0$ there. In other words, if $u\left(x, y_{0}\right)$ has a maximum at $x=x_{0}$, then $u\left(x_{0}, y\right)$ has a minimum at $y=y_{0}$. Thus, although it is not an extremum of $u(x, y)$ and $v(x, y),\left(x_{0}, y_{0}\right)$ is a minimax or saddle point of these two functions.

A further consequence of the holomorphy of $w(z)$ is that the curves $u(x, y)=$ constant and $v(x, y)=$ constant are the level curves for a conformal mapping and so are everywhere orthogonal. Thus, referring to Figure 6.1, we see that if we proceed along a curve $v(x, y)=$ constant, $u(x, y)$ will vary at its maximum rate. The curves $A B$ and $C D$ are the two curves passing through the saddle point that correspond to a constant value of $v(x, y)$. On one of them, $C D, u(x, y)$ increases as rapidly as possible; on the other, $A B, u(x, y)$ decreases as rapidly as possible.

Returning to our integral, we now recognize that condition (6.3.2) implies that $t=t_{0}$ is a saddle point of $\operatorname{Re}[z f(t)]$, while (6.3.3) and the requirement that $\operatorname{Re}[z f(t)]$ 


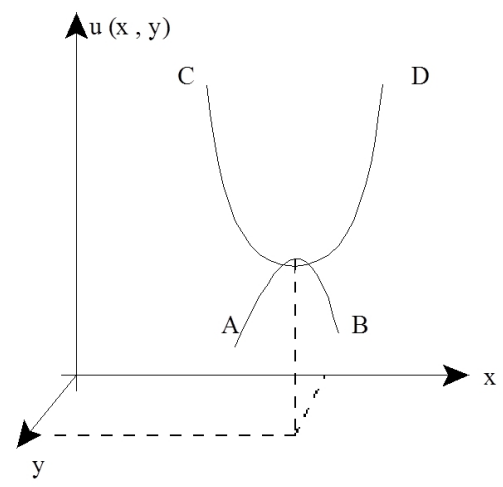

Figure 6.1: A saddle point

have a relative maximum at $t=t_{0}$ uniquely identifies $C_{0}$ as the counterpart of the curve $A B$. With this choice for $C_{0}, e^{z f(t)}$ goes to its end-point values by the steepest route possible, that is, by the path of steepest descent. Moreover, most of the value of the integral must come from the neighbourhood of $t=t_{0}$ since the modulus of the integrand is at a maximum there while its phase is roughly constant. (The phase is exactly constant if $g(t)$ is real.) This becomes increasingly true as $|z| \rightarrow \infty$ for the maximum becomes larger and the descent to the end-point values becomes steeper and steeper.

Assuming that we can deform our initial contour $C$ to coincide with $C_{0}$ without encountering any of the singularities of $f(t)$ or $g(t)$, we may rewrite (6.3.1) as

$$
F(z)=e^{z f\left(t_{0}\right)} \int_{C_{0}} e^{z\left[f(t)-f\left(t_{0}\right)\right]} g(t) d t
$$

where, by construction, $z\left[f(t)-f\left(t_{0}\right)\right]$ is real and negative for all $t$ on $C_{0}$ except at $t=t_{0}$ where it vanishes. This expression can be simplified in appearance by defining a real function $\tau(t)$ via the identity

$$
e^{i \arg z}\left[f(t)-f\left(t_{0}\right)\right] \equiv-\tau^{2}(t)
$$

We then have

$$
\begin{aligned}
F(z) & =e^{z f\left(t_{0}\right)} \int_{C_{0}} e^{-|z| \tau^{2}(t)} g(t) d t \\
& =e^{z f\left(t_{0}\right)} \int_{\Gamma_{0}} e^{-|z| \tau^{2}} g[t(\tau)] \frac{d t(\tau)}{d \tau} d \tau,
\end{aligned}
$$

where $\Gamma_{0}$ is the image of $C_{0}$ in the $\tau$ plane. We know that $\Gamma_{0}$ runs along the real axis and passes through the origin $\tau=0$. Moreover, while its end-points will vary from case to case, this variation is no barrier to an asymptotic evaluation of (6.3.6). For, as 
$|z|$ increases, the exponential in the integrand becomes sufficiently steep that the only significant contribution to $F(z)$ comes from a small segment of $\Gamma_{0}$ about $\tau=0$. Thus, at the risk of committing only a negligible error we may take the end-points of $\Gamma_{0}$ to be $\pm \infty$ and write

$$
F(z) \sim e^{z f\left(t_{0}\right)} \int_{-\infty}^{\infty} e^{-|z| \tau^{2}} g[t(\tau)] \frac{d t}{d \tau} d \tau .
$$

To complete the asymptotic evaluation of $F(z)$ we need now only express $g(t)$ and $\frac{d t}{d \tau}$ as functions of $\tau$. This is most appropriately done by means of power series expansions about $\tau=0$. Thus, leaving aside for the moment the problem of determining the coefficients in the series

$$
g[t(\tau)] \frac{d t}{d \tau}=\sum_{m=0}^{\infty} c_{m} \tau^{m},
$$

we substitute it into (6.3.7) to obtain

$$
F(z) \sim e^{z f\left(t_{0}\right)} \sum_{m=0}^{\infty} c_{m} \int_{-\infty}^{\infty} e^{-|z| \tau^{2}} \tau^{m} d \tau .
$$

The standard integral

$$
I_{m}=\int_{-\infty}^{\infty} e^{-\alpha \tau^{2}} \tau^{m} d \tau, \quad \alpha>0
$$

is known to assume the values

$$
I_{m}=\left\{\begin{array}{rrr} 
& \sqrt{\pi / \alpha}, & m=0 \\
\frac{(2 k-1) ! !}{2^{k} \alpha^{k}} & \sqrt{\pi / \alpha}, & m=2 k=2,4, \ldots . \\
0, & m=1,3,5, \ldots .
\end{array}\right.
$$

Thus, (6.3.9) can also be written as

$$
F(z) \sim e^{z f\left(t_{0}\right)} \sqrt{\frac{\pi}{|z|}}\left[c_{0}+\sum_{k=1}^{\infty} c_{2 k} \frac{(2 k-1) ! !}{2^{k}} \frac{1}{|z|^{k}}\right]
$$

which brings us to within a single step of our long sought-after asymptotic expansion for $F(z)$. That last step is to extract the dependence on $\arg z$ from the coefficients $c_{2 k}$ to obtain a series in $z$ rather than $|z|$. The result is

$$
F(z) \sim e^{z f\left(t_{0}\right)} \sqrt{\frac{\pi}{z}}\left[a_{0}+\sum_{k=1}^{\infty} a_{2 k} \frac{(2 k-1) ! !}{2^{k}} \frac{1}{z^{k}}\right]
$$

where the coefficients

$$
a_{2 k}=e^{i \frac{2 k+1}{2} \arg z} c_{2 k}, \quad k=0,1,2, \ldots .
$$


The explicit calculation of the coefficients $a_{2 k}$ is a tedious chore because it involves expressing $t-t_{0}$ as a power series in $\tau$ by inversion of equation (6.3.5) and then substituting into the power series expansion of $g(t)$ about $t=t_{0}$. The coefficient of the leading term is the sole exception; it is readily determined as follows.

From (6.3.8) we know that

$$
c_{0}=\left.\left\{g[t(\tau)] \frac{d t(\tau)}{d \tau}\right\}\right|_{\tau=0}=\left.g\left(t_{0}\right) \frac{d t(\tau)}{d \tau}\right|_{\tau=0} .
$$

Moreover, since $f^{\prime}\left(t_{0}\right)=0$, we have

$$
f(t)-f\left(t_{0}\right)=f^{\prime \prime}\left(t_{0}\right) \frac{\left(t-t_{0}\right)^{2}}{2 !}+\ldots
$$

and so

$$
\tau^{2}=-e^{i \theta} f^{\prime \prime}\left(t_{0}\right) \frac{\left(t-t_{0}\right)^{2}}{2 !}+\ldots
$$

where, for notational simplicity, we have set $\arg z=\theta$. Thus, inverting this series we find to lowest order that

$$
t-t_{0}=\frac{\sqrt{2} \tau}{\sqrt{e^{i(\pi+\theta)} f^{\prime \prime}\left(t_{0}\right)}}+\ldots
$$

and hence,

$$
\left.\frac{d t}{d \tau}\right|_{\tau=0}=\lim _{\tau \rightarrow 0} \frac{t-t_{0}}{\tau}=\frac{\sqrt{2}}{\sqrt{e^{i(\pi+\theta)} f^{\prime \prime}\left(t_{0}\right)}} .
$$

Substitution into (6.3.13) then yields

$$
c_{0}=\frac{\sqrt{2} g\left(t_{0}\right)}{\sqrt{e^{i(\pi+\theta)} f^{\prime \prime}\left(t_{0}\right)}} \quad \text { or, } \quad a_{0}=\frac{\sqrt{2} g\left(t_{0}\right)}{\sqrt{e^{i \pi} f^{\prime \prime}\left(t_{0}\right)}} .
$$

Therefore, retaining only the dominant term in the asymptotic expansion (6.3.11), we have

$$
F(z) \sim g\left(t_{0}\right) \sqrt{\frac{2 \pi}{e^{i \pi} f^{\prime \prime}\left(t_{0}\right)}} \frac{e^{z f\left(t_{0}\right)}}{\sqrt{z}} .
$$

To determine higher order terms we rewrite (6.3.14) as

$$
e^{-i \theta} \tau^{2}(t)=f\left(t_{0}\right)-f(t)=\sum_{m=2}^{\infty} \alpha_{m}\left(t-t_{0}\right)^{m}
$$

where $\alpha_{m}=-\frac{f^{(m)}\left(t_{0}\right)}{m !}, \quad m \geqslant 2$.

Then, since we seek a power series for $\left(t-t_{0}\right)$ about $\tau=0$, we set

$$
t-t_{0}=\sum_{m=0}^{\infty} \beta_{m} \tau^{m+1}
$$


and substitute this series into the right hand side of (6.3.17). Using

$$
\left(\sum_{k=0}^{\infty} \beta_{k} \tau^{k+1}\right)^{n}=\sum_{m=0}^{\infty} \gamma_{m} \tau^{m+n}
$$

where $\gamma_{0}=\beta_{0}^{n}, \gamma_{m}=\frac{1}{m \beta_{0}} \sum_{k=1}^{m}[k(n+1)-m] \beta_{k} \gamma_{m-k}, m \geqslant 1$, this yields

$$
\begin{aligned}
e^{-i \theta} \tau^{2}= & \alpha_{2} \tau^{2}\left[\beta_{0}^{2}+2 \beta_{1} \beta_{0} \tau+\left(\beta_{1}^{2}+2 \beta_{2} \beta_{0}\right) \tau^{2}+2\left(\beta_{2} \beta_{1}+\beta_{3} \beta_{0}\right) \tau^{3}\right. \\
& \left.\quad+\left(\beta_{2}^{2}+2 \beta_{1} \beta_{3}+2 \beta_{0} \beta_{4}\right) \tau^{4}+\ldots\right] \\
& +\alpha_{3} \tau^{3}\left[\beta_{0}^{3}+3 \beta_{1} \beta_{0}^{2} \tau+3\left(\beta_{1}^{2} \beta_{0}+\beta_{2} \beta_{0}^{2}\right) \tau^{2}\right. \\
& \left.\quad+\left(\beta_{1}^{3}+6 \beta_{2} \beta_{1} \beta_{0}+3 \beta_{3} \beta_{0}^{2}\right) \tau^{3}+\ldots\right] \\
& +\alpha_{4} \tau^{4}\left[\beta_{0}^{4}+4 \beta_{1} \beta_{0}^{3} \tau+\left(6 \beta_{1}^{2} \beta_{0}^{2}+4 \beta_{2} \beta_{0}^{3}\right) \tau^{2}+\ldots\right] \\
& +\alpha_{5} \tau^{5}\left[\beta_{0}^{5}+5 \beta_{1} \beta_{0}^{4} \tau+\ldots\right] \\
& +\alpha_{6} \tau^{6}\left[\beta_{0}^{6}+\ldots\right] \\
& +\ldots .
\end{aligned}
$$

Thus, equating coefficients of like powers of $\tau$ we obtain the following equations expressing $\beta_{0}, \beta_{1}, \beta_{2}, \ldots$ in terms of the $\alpha_{m}, m \geqslant 2$ :

$$
\begin{aligned}
& e^{-i \theta}=\alpha_{2} \beta_{0}^{2} \\
& 0=2 \alpha_{2} \beta_{1} \beta_{0}+\alpha_{3} \beta_{0}^{3} \\
& 0=\alpha_{2}\left(\beta_{1}^{2}+2 \beta_{2} \beta_{0}\right)+3 \alpha_{3} \beta_{1} \beta_{0}^{2}+\alpha_{4} \beta_{0}^{4} \\
& 0=2 \alpha_{2}\left(\beta_{2} \beta_{1}+\beta_{3} \beta_{0}\right)+3 \alpha_{3}\left(\beta_{1}^{2} \beta_{0}+\beta_{2} \beta_{0}^{2}\right)+4 \alpha_{4} \beta_{1} \beta_{0}^{3}+\alpha_{5} \beta_{0}^{5}
\end{aligned}
$$

Solving, we find

$$
\begin{aligned}
& \beta_{0}=\frac{e^{-i \theta / 2}}{\sqrt{\alpha_{2}}} \\
& \beta_{1}=-\frac{\alpha_{3}}{2 \alpha_{2}} \beta_{0}^{2} \\
& \beta_{2}=\left[\frac{5 \alpha_{3}^{2}}{8 \alpha_{2}^{2}}-\frac{\alpha_{4}}{2 \alpha_{2}}\right] \beta_{0}^{3} \\
& \beta_{3}=\left[-\frac{\alpha_{3}^{3}}{\alpha_{2}^{3}}+\frac{3 \alpha_{3} \alpha_{4}}{\alpha_{2}^{2}}-\frac{\alpha_{5}}{2 \alpha_{2}}\right] \beta_{0}^{4} \\
& \beta_{4}=\left[\frac{231 \alpha_{3}^{4}}{128 \alpha_{2}^{2}}-\frac{63 \alpha_{4} \alpha_{3}^{2}}{16 \alpha_{2}^{3}}+\frac{7 \alpha_{4}^{2}}{8 \alpha_{2}^{2}}+\frac{7 \alpha_{3} \alpha_{5}}{4 \alpha_{2}^{2}}-\frac{\alpha_{6}}{2 \alpha_{2}}\right] \beta_{0}^{5}
\end{aligned}
$$


Substitution of these coefficients into (6.3.18) and term-wise differentiation finally produces the power series about $\tau=0$ for $\frac{d t}{d \tau}$. To obtain the corresponding series for $g(t)$ requires still more tedious effort since we must substitute powers of (6.3.18) into

$$
g(t)=\sum_{m=0}^{\infty} \frac{g^{(m)}\left(t_{0}\right)}{m !}\left(t-t_{0}\right)^{m}
$$

and then collect coefficients of like powers of $\tau$. Fortunately, many interesting functions have integral representations with $g(t)=1$. In such cases the coefficients $c_{m}$ of equation (6.3.8) are given by

$$
c_{m}=(m+1) \beta_{m}, m=0,1,2, \ldots
$$

Combining this result with (6.3.21) and then (6.3.12), we find that the first three of the coefficients $a_{2 k}$ in the asymptotic expansion (6.3.11) of $F(z)$ are

$$
\begin{aligned}
& a_{0}=\alpha_{2}^{-1 / 2} \\
& a_{2}=\frac{3}{2} \alpha_{2}^{-3 / 2}\left[\frac{5}{4} \frac{\alpha_{3}^{2}}{\alpha_{2}^{2}}-\frac{\alpha_{4}}{\alpha_{2}}\right] \\
& a_{4}=\frac{5}{2} \alpha_{2}^{-5 / 2}\left[\frac{231}{64} \frac{\alpha_{3}^{4}}{\alpha_{2}^{4}}-\frac{63}{8} \frac{\alpha_{4} \alpha_{3}^{2}}{\alpha_{2}^{3}}+\frac{7}{4} \frac{\alpha_{4}^{2}}{\alpha_{2}^{2}}+\frac{7}{2} \frac{\alpha_{3} \alpha_{5}}{\alpha_{2}^{2}}-\frac{\alpha_{6}}{\alpha_{2}}\right]
\end{aligned}
$$

where we recall that $\alpha_{m}=-\frac{f^{(m)}\left(t_{0}\right)}{m !}, m \geqslant 2$.

Example: To illustrate the use of this formidable piece of mathematical machinery we shall determine the asymptotic expansion of $\Gamma(z+1)$. From Euler's definition, equation (5.2.5), we have

$$
\begin{aligned}
\Gamma(z+1) & =\int_{0}^{\infty} e^{-u} u^{z} d u, \quad \operatorname{Re} z>-1 \\
& =z^{z+1} \int_{0}^{\infty} e^{z(\ln t-t)} d t
\end{aligned}
$$

where we have set $u=z t$. Thus, in this case, $f(t)=\ln t-t$ and $g(t)=1$.

Differentiating $f(t)$ and setting the result equal to zero, $f^{\prime}\left(t_{0}\right)=\frac{1}{t_{0}}-1=0$, we see that $f(t)$ possesses only one saddle-point located at $t=t_{0}=1$. Hence, there is a single path of steepest descent which, because $f\left(t_{0}\right)=-1$, is uniquely defined by

$$
\operatorname{Im}[z f(t)]=-\operatorname{Im} z \quad \operatorname{Re}[z f(t)] \leqslant-\operatorname{Re} z
$$

We do not need a more detailed identification of the path because it is already clear that the initial contour of integration, the positive real axis, can be deformed to lie along it without encountering singularities of $f(t)$. Thus, it only remains to evaluate 
a few derivatives of $f(t)$ at $t=t_{0}=1$ and thence, to calculate the asymptotic series coefficients $a_{0}, a_{2}, \ldots$. The first of these tasks is easily accomplished: $f^{(m)}(1)=$ $(-1)^{m-1}(m-1)$ ! and so, $\alpha_{m}=\frac{(-1)^{m}}{m}, m \geqslant 2$. Substituting into (6.3.23) we then find

$$
a_{0}=2^{1 / 2}, \quad a_{2}=\frac{1}{12} 2^{3 / 2}, \quad a_{4}=\frac{1}{864} 2^{5 / 2} .
$$

Therefore, by equation (6.3.11), the asymptotic expansion of $\Gamma(z+1)$ is

$$
\Gamma(z+1) \sim \sqrt{2 \pi} z^{z+1 / 2} e^{-z}\left[1+\frac{1}{12} \frac{1}{z}+\frac{1}{288} \frac{1}{z^{2}}+\ldots\right]
$$

which is known as Stirling's approximation.

Born in Scotland in 1692, James Stirling was a contemporary of and correspondent with such notable mathematicians as Euler, DeMoivre and Newton. His most important work is a treatise, Methodus Differentialis, published in London in 1730. It contains the asymptotic formula for $n$ ! to which his name is attached. Stirling was believed to have Jacobite sympathies which was an impediment to academic advancement in Hanoverian Britain. Obliged to work as a mine manager, his mathematical output declined. Nevertheless, Euler secured his election to the Royal Academy of Berlin in 1746 just as Newton had arranged for his election to the Royal Society in 1726. Stirling died in Edinburgh in 1770.

We shall have occasion to use the method of steepest descent several times in subsequent Chapters, particularly when it comes time to discuss the properties of Bessel functions. However, what really commends it to physicists are its direct applications in modern physics. Important examples are the evaluation of partition functions in statistical mechanics and of generating functionals in the path integral formalism of quantum mechanics and quantum field theory. 